\title{
Erratum to: Possible involvement of heterotrimeric G-protein signaling in Al-induced secretion of organic acid anions in Arabidopsis and rye
}

\author{
Yao Yan Li • Xin Lian Tang • Lei Geng Yang • \\ Yong Xiong $\mathbf{Y u} \cdot$ Xiao Feng Li
}

Published online: 13 December 2014

(C) Springer International Publishing Switzerland 2014

\section{Erratum to: Plant Soil (2014) \\ DOI 10.1007/s11104-014-2325-0}

The published paper unfortunately contained a mistake. The first author (Yao Yan Li ) has been misspelled as Yao Yang Li and the details has been corrected in the author group.

The online version of the original article can be found at http:// dx.doi.org/10.1007/s11104-014-2325-0.

\section{Y. Y. Li $\cdot$ X. L. Tang $\cdot$ L. G. Yang $・$ X. F. Li $(\bowtie)$}

State Key Laboratory for Conservation and Utilization of Subtropical Agro-bioresources, College of Agriculture, Guangxi University, Nanning 530005, China

e-mail: 1xf@gxu.edu.cn

Y. Y. Li

e-mail: liyaoyan08@163.com

X. L. Tang

e-mail: txl@gxu.edu.cn

L. G. Yang

e-mail: 176630911@qq.com

Y. Y. Li

Guangxi University of Chinese Medicine,

Nanning 530001, China

Y. X. Yu

College of Animal Science and Technology, Southwest

University,

Beibei Chongqing 400716, China

e-mail: yuyongxiong8@126.com 